\title{
Interspecies communication in bacteria
}

\author{
Michael J. Federle and Bonnie L. Bassler \\ Department of Molecular Biology, Princeton University, Princeton, New Jersey, USA
}

\begin{abstract}
Until recently, bacteria were considered to live rather asocial, reclusive lives. New research shows that, in fact, bacteria have elaborate chemical signaling systems that enable them to communicate within and between species. One signal, termed AI-2, appears to be universal and facilitates interspecies communication. Many processes, including virulence factor production, biofilm formation, and motility, are controlled by AI-2. Strategies that interfere with communication in bacteria are being explored in the biotechnology industry with the aim of developing novel antimicrobials. AI-2 is a particularly attractive candidate for such studies because of its widespread use in the microbial kingdom.
\end{abstract}

J. Clin. Invest. 112:1291-1299 (2003). doi:10.1172/JCI200320195.

\section{Species-specific cell-cell communication}

Quorum sensing is a process in which bacteria monitor their cell-population density by measuring the concentration of small secreted signal molecules, called autoinducers. As a population of quorum-sensing bacteria grows, the individual organisms produce and secrete the autoinducers into the extracellular environment. Thus, the concentration of external autoinducer is correlated with cell-population density. By monitoring the extracellular autoinducer concentration, the bacteria can "count" one another and alter target-gene expression accordingly (1). Quorum-sensing systems can be divided into two paradigmatic classes: LuxI/LuxR-type quorum-sensing systems in Gram-negative bacteria and oligopeptide/two-component-type quorum-sensing circuits in Gram-positive bacteria (Figure 1).

In Gram-negative bacteria, the LuxI-like proteins are the enzymes responsible for producing specific acylhomoserine lactone (AHL) autoinducers (Figure 2a). Each species of Gram-negative bacteria produces a unique AHL or a unique combination of AHLs (if it possesses more than one LuxI-type protein). As a result, only the members of the same species recognize and respond to it. AHL detection and subsequent alteration of downstream gene expression are mediated by a cognate LuxR protein (Figure 1a) (2). Following LuxIdirected AHL synthesis, the autoinducer freely diffuses into and out of the cell (3). Thus, the external AHL

\footnotetext{
Address correspondence to: Bonnie L. Bassler, Department of Molecular Biology, Princeton University, Princeton, New Jersey 08544-1014, USA. Phone: (609) 258-2857; Fax: (609) 258-6175; E-mail: bbassler@molbio.princeton.edu.

Conflict of interest: The authors have declared that no conflict of interest exists.

Nonstandard abbreviations used: acyl-homoserine lactone (AHL); autoinducing peptide (AIP); acetyltransferase-repressing factor (AR factor); Rhomboid (RHO); $S$-adenosylmethionine (SAM); 4,5-dihydroxy-2,3-pentanedione (DPD); LuxS-regulated (Lsr).
}

concentration is equivalent to the internal (cytoplasmic) AHL concentration, and it increases proportionally with increasing cell density. Once a threshold AHL concentration is achieved, the autoinducer can be bound by its cytoplasmic counterpart, the LuxR protein (Figure 1a). Upon interaction with a cognate AHL, a LuxR-type protein binds specific DNA promoter elements and activates downstream target-gene transcription (4). AHL-LuxR interactions are remarkably specific, and thus there appears to be extremely limited cross-talk in mixed populations of bacteria that use AHL-LuxR-mediated cell-cell communication.

Over 70 species of Gram-negative bacteria have recently been identified that use analogous LuxI/LuxR quorum-sensing systems to control a wide range of processes (1). These systems are covered in more depth in other reviews in this Perspective series.

In stark contrast to Gram-negative bacteria, Gram-positive bacteria have never been shown to use AHL-mediated quorum sensing. Instead, Gram-positive bacteria make and transport oligopeptide autoinducers into their environment (Figure 1b). These oligopeptides, also known as autoinducing peptides (AIPs), typically consist of 5-17 amino acids, sometimes containing unusual side chain modifications (5). Figure $2 \mathrm{~b}$ shows a sample of Gram-positive AIPs. In further contrast to AHL signaling, the bacterial cell membrane is not permeable to AIPs, necessitating dedicated cell-surface oligopeptide transporters to facilitate AIP secretion into the extracellular environment. Detection of AIPs is mediated by twocomponent sensory-transduction systems.

Two-component systems exist in a wide variety of Gram-negative and Gram-positive bacteria. These circuits are responsible for detecting fluctuations in a vast assortment of extracellular cues and transducing that sensory information into the cell to appropriately modulate gene expression in response to a changing environment (6). 


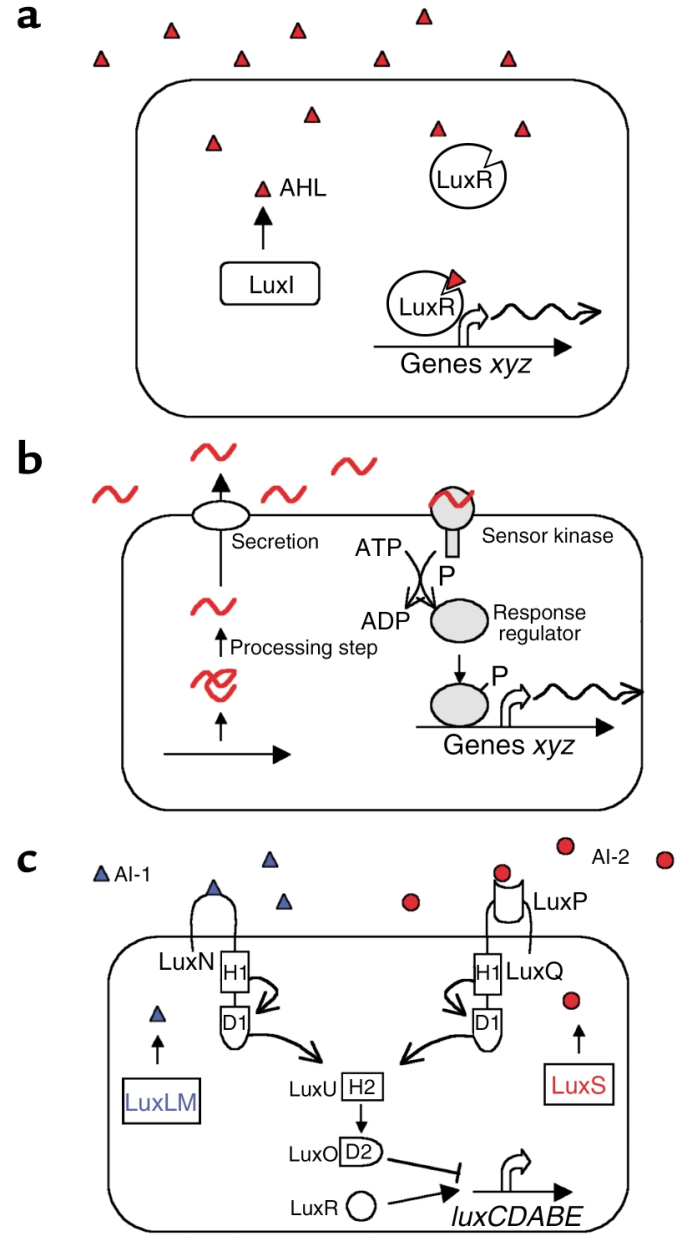

All bacterial two-component systems function by a conserved phosphorylation cascade involving a sensor kinase and response regulator pair. The sensor kinases contain invariant histidine residues, and the response regulators contain invariant aspartic acid residues; these

\section{Figure 1}

Three canonical quorum-sensing circuits in bacteria. (a) In Gramnegative bacteria, AHLs (red triangles) are produced by Luxl-like proteins and are detected by LuxR-type proteins. AHLs freely diffuse across the cell membrane and increase in concentration in the environment in proportion to cell growth. LuxR-type proteins, when bound to cognate autoinducers, bind specific promoter DNA elements and activate transcription of target genes (xyz). (b) Gram-positive bacteria synthesize oligopeptides (red wavy lines) that are typically modified at specific amino acids and are actively secreted. Detection occurs via a two-component signal transduction circuit, leading to the phosphorylation of a response regulator protein, which can bind promoter DNA and regulate transcription of target genes $(x y z)$. (c) Quorum sensing in Vibrio harveyi. Two parallel twocomponent systems detect $\mathrm{Al}-1$ (blue triangles), an $\mathrm{AHL}$ synthesized by LuxLM, and Al-2 (red circles), a furanosyl borate diester, which is synthesized by LuxS. In the absence of autoinducer, the sensors act as kinases and autophosphorylate at a conserved histidine residue, $\mathrm{H} 1$, and the phosphate is transferred to a conserved aspartate residue, $\mathrm{D} 1$, in the response regulator domain. Phosphate is sequentially transferred to the conserved histidine $(\mathrm{H} 2)$ of the phosphotransferase LuxU and then to the conserved aspartate (D2) of the response regulator LuxO. Phospho-LuxO indirectly represses transcription of luxCDABE, the enzymes encoding luciferase. Binding of the autoinducers by LuxN and LuxPQ leads to the dephosphorylation of LuxU and LuxO. Dephosphorylation of LuxO relieves repression of luxCDABE. A transcriptional activator called LuxR (not similar to LuxR in a) is required for expression of luxCDABE.

residues are the sites of phosphorylation (7). The modular domain organization of the two-component circuits can vary, as these circuits can be composed of two, three, or four protein partners. Some canonical twocomponent circuits are shown in Figure 3. The final component of the circuit (the response regulator) is usually a DNA-binding protein that, when phosphorylated, alters target-gene expression (6). In the present context, the AIPs are the ligands for the two-component sensor kinases, and the phosphorylation cascades relay

\section{Figure 2}

Structures of different bacterial autoinducers. (a) Examples of AHL autoinducers of some Gram-negative bacteria. (b) A selection of Gram-positive AIP autoinducers. The asterisk above the tryptophan (W) of ComX indicates a posttranslational isoprenylation of the peptide. The AIP molecules of Staphylococcus aureus are shown with the thioester bridge linking the indicated amino acid residues. The numbering I-IV refers to the $S$. aureus group classification. (c) Al-2 of Vibrio harveyi is a furanosyl borate diester, as determined by co-crystallization with the $V$. harveyi $\mathrm{Al}-2$ receptor LuxP. a Vibrio fischeri/LuxI<smiles>CCCC(=O)CC(=O)NC1CCOC1=O</smiles>

Pseudomonas aeruginosa/Lasl<smiles>CCCCCCCCCC(=O)CC(=O)NC1CCOC1=O</smiles>

Pseudomonas aeruginosa/Rh1I<smiles>CCCC(=O)NC1CCOC1=O</smiles>

Agrobacterium tumefaciens/Tral<smiles>CCCCCC(=O)CC(=O)NC1CCOC1=O</smiles>

Vibrio harveyi/LuxLM<smiles>[M]C(C)CC(=O)NC1CCOC1=O</smiles>

b ERGMT ADPITRQWैGD EMRLSKFFRDFILQRKK ComX (Bacillus subtilis) pneumoniae) AIPs (Staphylococcus aureus)

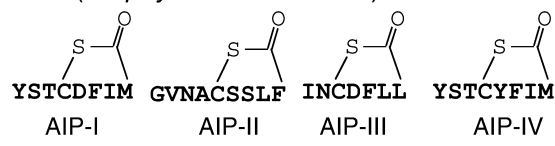<smiles>C[C@]12OCC(O)[C@]1(O)O[Pb](O)(O)O2</smiles> 


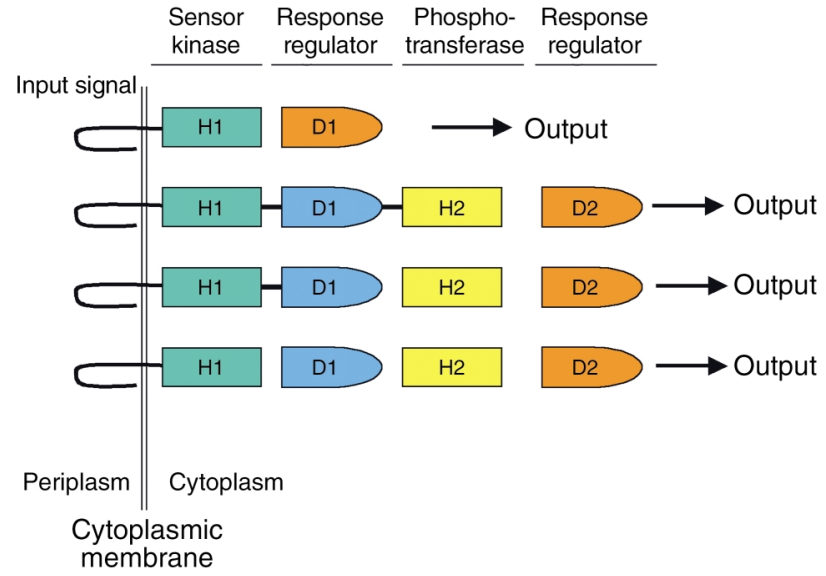

Figure 3

Modular organization of two-component signal transduction cascades in bacteria. Bacteria typically detect changes in their environment using two-component phosphorelay systems. Information is detected by the first protein component, which is usually a membrane-spanning sensor-histidine kinase (green). Phosphate is transferred to the response regulator protein that is responsible for controlling the output (red). The sensor protein is autophosphorylated on a conserved histidine residue $(\mathrm{H} 1)$. Phosphate is transferred to a conserved aspartate residue (D1) on the response regulator. Twocomponent systems often include additional response regulator and phosphotransferase proteins (blue and yellow domains, respectively) that contain conserved aspartate and histidine residues. The modular domain organization of the two-component circuits can vary, as these circuits can be composed of two, three, or four protein partners.

information about the population density into the cell. Many Gram-positive AIP/two-component cell-cell communication systems are known. As in AHL-regulated cell-cell signaling, the differences in the ligands, in this case the differences in the amino acid sequences of the AIPs, confer exquisite species specificity to these communication circuits, since each two-component sensor kinase is highly selective for a response to its cognate AIP and no other (see Figure 2b).

AIP systems are thoroughly covered in other reviews in this Perspective series. We describe one well-studied circuit here. The pathogen Staphylococcus aureus uses a modified AIP containing a thiolactone ring (Figure 2b) to regulate virulence factor production in response to increases in cell-population density (8-10). Specifically, late in exponential growth, detection of the accumulation of a specific AIP causes $S$. aureus to downregulate surface-associated factors that promote attachment and to upregulate secreted proteins including a variety of toxins (11). Presumably, the reciprocal control of the genes specifying attachment and toxin secretion allows $S$. aureus to proceed from initial surface colonization to invasion of the host. S. aureus AIP signaling functions at the subspecies level because the AIP of one group of $S$. aureus strains, while initiating its own virulence cascade, inhibits the initiation of the virulence cascades of all other groups of $S$. aureus strains (10). S. aureus can be grouped into four classes according to the AIP produced (Figure 2b). The finding that $S$. aureus AIPs specif- ically activate genes in one group of strains while specifically inhibiting those same genes in another group of strains suggests that the different groups of $S$. aureus represent intermediate steps in the process of evolution of new species. This remarkable feature of $S$. aureus cellcell communication is further discussed below.

A non-canonical quorum-sensing system exists in Providencia stuartii. This Gram-negative bacterium is a urinary-tract pathogen in humans. An autoinducer termed acetyltransferase-repressing (AR) factor is required for quorum-sensing control of genes specifying enzymes involved in the acylation of peptidoglycan and aminoglycosides (12). Although AR factor has not been completely characterized, preliminary analysis suggests that it has peptide-like biochemical properties, which is the first indication that Gram-negative bacteria, like Grampositive bacteria, use secreted peptides for intercellular communication. Genetic analysis has shown that release of the AR factor requires a gene called aarA, which, surprisingly, encodes a protein that is homologous to the intramembrane serine protease Rhomboid (RHO) of Drosophila melanogaster $(13,14)$. In $D$. melanogaster, RHO is required for the cleavage of EGF. Cleavage liberates EGF from the membrane into the extracellular environment, where it functions as the ligand in EGFR-mediated cell-cell signaling (15). Sequence analysis suggests that highly conserved RHO homologs exist in at least 50 bacterial genomes. Evidence indicating that these proteases perform analogous signaling functions in prokaryotes and eukaryotes comes from the finding that the P. stuartii RHO-like gene aarA is functionally interchangeable with rhomboid of D. melanogaster (14). Specifically, introduction of aarA rescues wing vein and compound eye development in various $D$. melanogaster rhomboid mutants, and conversely, the $D$. melanogaster rbo- 1 gene substitutes for aarA in P. stuartii and facilitates the processing and release of the $P$. stuartii quorum-sensing AR factor into the growth medium. These studies suggest a common origin for some prokaryotic and eukaryotic cell-cell signaling systems.

The quorum-sensing systems described above promote intraspecies communication, meaning that only the species of bacteria that produces the autoinducer can detect and respond to it. Highly specific intraspecies communication circuits may be important for bacteria that reside in niches occupied by multiple species of bacteria, such as the human gastrointestinal tract. In these environments, intraspecies cell-cell communication could enable each individual species to gauge its own numbers and to coordinate particular behaviors while immersed in an environment potentially containing an array of molecules with similar chemical structures. The exquisite species specificity built into these quorumsensing systems might be critical for allowing bacteria to discriminate self from other and signal from noise.

\section{Interspecies cell-cell communication}

While species-specific quorum sensing apparently allows recognition of self in a mixed population, it 


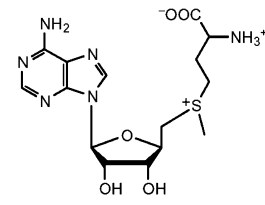

SAM

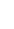

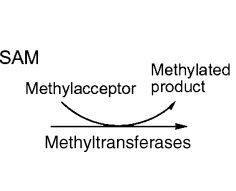

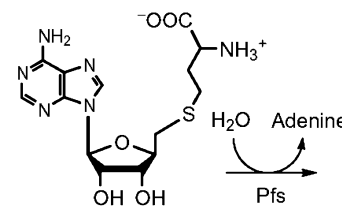

$\mathrm{SAH}$

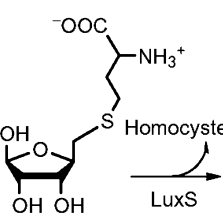

$\mathrm{SRH}$

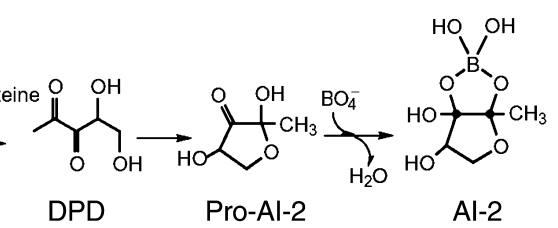

Al-2

\section{Figure 4}

Biosynthesis of Al-2. Utilization of SAM as a methyl donor in cellular processes yields S-adenosylhomocysteine (SAH). The enzyme Pfs converts SAH to S-ribosylhomocysteine (SRH). LuxS is responsible for the conversion of SRH to homocysteine and DPD. DPD is predicted to spontaneously rearrange into various furanones. The furanone predicted to lead to the formation of $\mathrm{V}$. harveyi Al-2 is the only one shown and is termed pro-Al-2. Borate adds to pro-Al-2 to form the active signaling molecule Al- 2 .

seems likely that in such situations, bacteria also need a mechanism or mechanisms to detect the presence of other species. Additionally, it is conceivable that it is useful for bacteria to have the ability to calculate the ratio of self to other in mixed populations, and in turn, to specifically modulate behavior based on fluctuations in this ratio. Evidence for the existence of these types of complex mechanisms of cell-cell communication came first from studies of the Gram-negative bioluminescent shrimp pathogen Vibrio harveyi. Genetic analysis of the $V$. harveyi quorum-sensing circuit revealed that this bacterium uses two different autoinducer signals to regulate light production and a number of other target outputs (16-18). In this regulatory circuit, it appears that one signal is used for intraspecies communication and the second signal is used for interspecies communication.

Interestingly, the $V$. harveyi quorum-sensing circuit is composed of functions found in typical Gram-negative and typical Gram-positive quorum-sensing systems (Figure 1c). Similar to other Gram-negative bacteria, $V$. harveyi produces and responds to an AHL-type autoinducer termed AI-1 (Figure 2a) (19). In contrast to Gram-negative bacteria but analogous to Gram-positive bacteria, autoinducer-mediated signal relay in $V$. harveyi is carried out by two-component signal transduction proteins. Additionally, $V$. harveyi possesses a novel signaling molecule, denoted AI-2 $(20,21)$. Genetic and biochemical evidence suggests that AI-1 mediates intraspecies communication and AI- 2 mediates interspecies cell-cell communication.

In $V$. harveyi, the AHL signal AI- 1 is synthesized by the protein LuxLM (Figures 1c and 2a). LuxLM shares no homology with the LuxI family of AHL autoinducer synthases; however, the LuxLM protein performs biochemical tasks identical to those performed by LuxItype enzymes (19). These enzymes carry out reactions to ligate the acyl moiety from an acyl-acyl carrier protein (acyl-ACP) to the homocysteine moiety of S-adenosylmethionine (SAM). AI-1 is detected by the two-component hybrid sensor kinase-response regulator protein LuxN (19). The second $V$. harveyi signal, AI-2, is synthesized by an enzyme called LuxS $(20,22)$. AI- 2 is a furanosyl borate diester (Figure 2c) (21). Like AHLs, AI- 2 is derived from SAM, in this case in three enzymatic steps (Figure 4; also, see below) $(20,23)$. Detec- tion of AI-2 requires two proteins: LuxP and LuxQ (17). LuxP is a soluble periplasmic protein resembling sugarbinding proteins such as RsbB of Escherichia coli, which recognizes ribose. LuxP is the AI-2-binding protein, and the LuxP-AI-2 complex interacts with the second protein required for detection, LuxQ. LuxQ is similar to LuxN and is a hybrid two-component protein containing both sensor kinase and response regulator modules. Sensory information from both LuxN and LuxPQ is transduced to the phosphotransferase protein LuxU, and LuxU transmits the signal to the downstream response regulator LuxO (Figure 1c) (24).

The $V$. harveyi quorum-sensing circuit functions as follows. Under conditions of low cell density (i.e., in the absence of autoinducers), LuxN and LuxQ act as kinases. Analogous to all other two-component sensors, they autophosphorylate on conserved histidine residues. Phosphate is sequentially transferred to the conserved aspartate residue on the response regulator modules of the hybrid proteins, and then to a conserved histidine on LuxU, and finally to the conserved aspartate on LuxO. Phospho-LuxO is indirectly responsible for repression of the luxCDABE operon, which encodes the luciferase enzymes necessary for light production (25). At high cell density (i.e., when the autoinducers are present), interactions of the signals with LuxN and LuxPQ cause the sensors to switch from kinase mode to phosphatase mode. Phosphate flows backward through the circuit traveling via the same histidine and aspartate residues used in the forward direction. The net result is the dephosphorylation and inactivation of LuxO, leading to derepression of $\operatorname{lu} x C D A B E$. Under this condition, a transcriptional activator called LuxR (not similar to other LuxR-type proteins) induces transcription of luxCDABE and light production (26-28).

Production and detection of AI-1 appear to be unique to $V$. harveyi and the closely related species Vibrio parahaemolyticus (29). However, luxS is present in the genomes of a wide variety of Gram-negative and Grampositive bacteria (Table 1), and every bacterium whose genome contains a functional $\operatorname{lu} x S$ gene has been shown to be capable of producing an activity that can be detected by an AI-2-specific $V$. harveyi reporter strain. Together, these two findings raise the possibility that unlike AHL- and AIP-mediated species-specific communication, AI-2 is a more universal signal that 
Table 1

Bacteria that possess luxS genes

$\begin{array}{lll}\text { Actinobacillus pleuropneumoniae } & \text { Helicobacter pylori } & \text { Salmonella paratyphi } \\ \text { Bacillus anthracis } & \text { Klebsiella pneumoniae } & \text { Salmonella typhi } \\ \text { Bacillus cereus } & \text { Lactobacillus gasseri } & \text { Salmonella typhimurium } \\ \text { Bacillus halodurans } & \text { Lactobacillus plantarum } & \text { Shigella flexneri } \\ \text { Bacillus subtilis } & \text { Lactococcus lactis } & \text { Sinorhizobium meliloti } \\ \text { Bifidobacterium longum } & \text { Leuconostoc mesenteroides } & \text { Staphylococcus aureus } \\ \text { Borrelia burgdorferi } & \text { Leuconostoc oenos } & \text { Staphylococcus epidermidis } \\ \text { Campylobacter jejuni } & \text { Listeria innocua } & \text { Streptococcus agalactiae } \\ \text { Clostridium acetobolyticum } & \text { Listeria monocytogenes } & \text { Streptococcus gordonii } \\ \text { Clostridium difficile } & \text { Neisseria gonorrhoeae } & \text { Streptococcus mutans } \\ \text { Clostridium perfringens } & \text { Neisseria meningitidis } & \text { Streptococcus pneumoniae } \\ \text { Deinococcus radiodurans } & \text { Oceanobacillus iheyensis } & \text { Streptococcus pyogenes } \\ \text { Enterococcus faecalis } & \text { Oenococcus oeni } & \text { Vibrio anguillarum } \\ \text { Enterococcus faecium } & \text { Pasteurella multocida } & \text { Vibrio cholerae } \\ \text { Escherichia coli } & \text { Pasteurella multocida } & \text { Vibrio harveyi } \\ \text { Haemophilus ducreyi } & \text { Porphyromonas gingivalis } & \text { Vibrio parahemolyticus } \\ \text { Haemophilus influenzae } & \text { Proteus mirabilis } & \text { Vibrio vulnificus } \\ \text { Haemophilus somnus } & \text { Salmonella enterica } & \text { Yersinia pestis } \\ \text { Helicobacter hepaticus } & & \end{array}$

The BLAST search algorithm available on the National Center for Biotechnology Information website (http://www.ncbi.nlm.nih.gov/BLAST/) was used to identify homologues of the $V$. harveyi luxS gene in bacterial species whose genomes have been sequenced. Al-2 production has been observed in many additional species whose genomes have not been sequenced, indicating that they too have a luxS gene.

could promote interspecies bacterial communication. Furthermore, the finding that bacteria detect and integrate information supplied by multiple chemical signals with different origins suggests that the bacteria use a combinatorial approach to distinguish between one another that is reminiscent of the molecular mechanism underlying odor detection and differentiation in higher organisms. It is also possible that bacteria possessing multiple AHL or AIP circuits use combinatorial means to process the information provided by these intraspecies-specific signals.

\section{Biosynthesis of Al-2}

The biosynthetic pathway leading to AI-2 production has been determined (20). LuxS is the third enzyme required in a three-step conversion of SAM to 4,5-dihydroxy-2,3-pentanedione (DPD) (Figure 4) (20). Utilization of SAM as a methyl donor in vital cellular processes yields the toxic metabolic intermediate $S$-adenosylhomocysteine (SAH). The enzyme Pfs rapidly hydrolyzes SAH to adenine and S-ribosylhomocysteine (SRH). In the third step, SRH is converted to DPD and homocysteine by LuxS. However, DPD is unstable and is proposed to spontaneously rearrange to form a variety of furanones. Structural analysis of the V. harveyi AI-2-binding protein LuxP in complex with the active AI-2 ligand showed that the signaling molecule is a furanosyl borate diester (Figure 2c) (21). This result indicates that borate is added to one of the furanone products formed from DPD (termed pro-AI-2 in Figure 4) to form the final $V$. harveyi $\mathrm{AI}-2$ signal molecule.

The characterization of AI-2 is significant for several reasons. First, because of its widespread nature and because it is unlike other types of known autoinducer, AI-2 defines the third major category of autoinducer. Second, to date, AI-2 is the only autoinducer that is made and recognized (as discussed below) by a broad range of bacteria, suggesting that AI- 2 provides the first known molecule that facilitates communication between different bacterial species. At present, only the structure of the $V$. harveyi AI- 2 has been determined. However, the finding that the highly reactive and unstable molecule DPD is an intermediate in the signal generation process raises the possibility that DPD undergoes other rearrangements to form a variety of cyclic compounds that react with borate and/or other available reactive moieties to form signaling molecules with structures that differ from that of V. harveyi $\mathrm{AI}-2$. This would mean that, like the AHLs and the AIPs, AI-2 may be a family of related molecules. Finally, it was unexpected, yet intriguing, that AI- 2 contains a boron atom. Boron has long been known to be an essential element in many organisms, but for unknown reasons. While it remains unclear why boron is critical for AI-2 signaling in $V$. harveyi, the addition of borate to DPD drives the conversion of DPD through pro-AI-2 to the stable borate-containing active AI-2 molecule. In support of these chemical findings, addition of borate to $l u x S^{+} V$. harveyi greatly enhances LuxPQ-dependent light production (21). Boron is widely available in the biosphere, and these findings suggest a critical biological signaling role for this element. Structural analyses of the form of AI-2 that is produced by and active in other bacteria should address whether boron is required in every case of AI-2dependent signaling, and, if so, why.

AI-2 facilitates interspecies communication, which we presume enhances the cooperation and therefore the survival of most or all of the bacterial species in a mixed community. If future studies reveal that AI- 2 is a single chemical entity, then AI- 2 can only designate that a particular environment contains "other" species, but it cannot supply information about the identities of these species. If this is the case, then, presumably, additional as-yet undiscovered molecules are produced by the various members of bacterial communities that aid in species identification. If, on the other hand, AI-2 is a family of molecules derived from a single precursor (DPD), then the particular blend of AI-2-related molecules present in a given environment could contain all the information required to specify the identities of the inhabitants.

\section{Al-2-controlled behaviors}

Not only is AI-2 produced by a wide variety of bacteria, it is also detected by many or possibly all of them. A vari- 
ety of outputs are controlled by AI- 2 in different bacteria, indicating that, as in AHL and AIP signaling, $\mathrm{AI}-2$ signaling has been adapted by the different bacteria that use it to influence a variety of niche-specific behaviors (Table 2; for a review of these behaviors see ref. 30). Examples include enterohemorrhagic E. coli (EHEC) and enteropathogenic $E$. coli (EPEC), which require LuxS to control the production of a type III secretion apparatus that enables tight adherence of the bacteria to the intestinal epithelia and leads to injection of proteins that direct the formation of attachment and effacing lesions (31). DNA microarray hybridization analysis in E. coli has suggested that $\mathrm{AI}-2$ signaling affects a large regulon consisting of $5-10 \%$ of the E. coli genome $(31,32)$.

In Salmonella typhimurium, $\mathrm{AI}-2$ is produced and released during exponential growth and is subsequently transported back into the bacteria via the Lsr (Lux $\underline{\text { S- }}$ regulated) $\mathrm{ABC}$ transporter $(33,34)$. AI-2 induces transcription of the lsrACDBFGE operon, the first four genes of which encode the Lsr transport apparatus. The downstream genes in the lsr operon along with two linked genes, $l s r K$ and $l s r R$, are required for the phosphorylation and processing of internalized AI-2. Transport and processing of AI-2 in S. typhimurium are hypothesized to be required for eliminating the quorum-sensing signal, for conveying the signal to an internal detector, and/or for scavenging boron.

Vibrio cholerae uses AI-2 together with its species-specific AI-1, cholerae AI-1 (CAI-1), to control virulence gene expression and biofilm formation. In contrast to most other pathogens, which use quorum sensing to induce the expression of virulence factors and biofilmformation genes at high cell density, $V$. cholerae uses quorum sensing to induce genes responsible for these traits at low cell density and to repress these factors at high cell density $(35,36)$. Specifically, at low cell density, in the absence of autoinducer, genes required for biofilm formation and the production of cholera toxin, toxin-coregulated pilus, and approximately 70 other virulence-associated genes are expressed (37). The protein products are required for adhesion of $V$. cholerae to intestinal epithelial cells and for the bacterium to cause the diarrheal disease cholera. Autoinducer accumulation at high cell density inhibits the expression of these same genes. Interestingly, at high cell density, while inhibiting the virulence regulon, quorum sensing induces the expression of a gene called hap, which encodes the Hap protease. This protease is suggested to act as a "detachase" that allows $V$. cholerae to be released from the intestine and to re-enter the environment (38). Reciprocal quorum-sensing regulation of virulence factors and the Hap "detachase" is consistent with the natural ecology of $V$. cholerae as a self-limiting pathogen that persists in the environment.

Gram-positive bacteria also use AI-2 as a signal. For example, two major virulence factors are affected by AI-2 in Streptococcus pyogenes. Specifically, in a luxS mutant, the secretion and processing of a virulenceassociated cysteine protease is greatly decreased, while the transcription of the gene encoding the virulence factor streptolysin $S$ is increased tenfold (39). These initial results suggest that, similar to what has been shown in V. cholerae, in S. pyogenes, AI-2 controls a complex regulatory network involving both positive and negative regulation. Another Gram-positive bacterium, Clostridium perfringens, which causes gas gangrene, uses AI-2 to control toxin production. In a $l u x S$ mutant, $\alpha$-, $\kappa$-, and $\theta$-toxin production is significantly decreased (40). However, AI-2 appears only to affect the transcription of $p f o A$, the gene encoding $\theta$-toxin.

\section{Table 2}

LuxS/Al-2-regulated behaviors

\begin{tabular}{llc}
\hline Species & Function & References \\
Actinobacillus actinomycetemcomitans & Virulence, iron acquisition & 52,53 \\
Borrelia burgdorferi & Pleiotropic protein expression & 54 \\
Campylobacter jejuni & Motility & 55 \\
Clostridium perfringens & Toxin production & 40 \\
Escherichia coli W3110 & Cell division, motility, metabolism & 32 \\
Escherichia coli, EHEC and EPEC & Virulence, type III secretion & 31,56 \\
Neisseria meningitidis & Bacteremic infection & 57 \\
Photorhabdus luminescens & Antibiotic production (carbapenem) & 58 \\
Porphyromonas gingivalis & Biofilm formation, heme acquisition, protease production & $44,59,60$ \\
Salmonella typhi & Biofilm formation & 61 \\
Salmonella typhimurium & ABC transporter expression & 33,34 \\
Shigella flexneri & Transcription factor associated with virulence & 62 \\
Streptococcus mutans & Biofilm formation & 63 \\
Streptococcus pneumoniae & Virulence & 64 \\
Streptococcus pyogenes & Virulence factor expression & 39 \\
Vibrio cholerae & Virulence factor expression & 35,37 \\
Vibrio harveyi & Luminescence, protease production, type III secretion, colony morphology, & $17,18,45$ \\
Vibrio vulnificus & siderophore production & 65
\end{tabular}


This result indicates that regulation of $\alpha$ - and $\kappa$-toxins occurs at a post-transcriptional level.

AI-2 has also proved important in the organization of bacteria into structures called biofilms. Bacterial biofilms can be composed of single or multiple species. In these communities, bacteria live adhered to surfaces and the biofilm inhabitants cooperate in the production of an ECM that leads to the formation of a complex architecture containing structures including "pillars" and "mushrooms." These structures are punctuated by aqueous channels that provide hydration while removing wastes from the different regions of the biofilm (41). It is clear in both monospecies and mixed-species biofilms that bacteria residing in different regions exhibit discrete patterns of gene expression. This finding indicates that the tasks required for the development and maintenance of a productive biofilm are not distributed uniformly. Rather, it appears that the expression of specific subsets of biofilm genes is allocated to different participants in the community. Biofilms are ubiquitous in nature and can be found in the human body in the oral cavity, in the lungs of cystic fibrosis patients, and on the surfaces of catheters and implanted prosthetic devices (41). AHL autoinducers, most notably those of Pseudomonas aeruginosa, are known to be required for biofilm maturation, reflecting that intraspecies cell-cell communication is a vital step in the proper development of these complex communities $(42,43)$. Recent investigations also indicate that AI-2-mediated interspecies cell-cell communication is necessary to coordinate biofilm production. The role of AI-2 in biofilm formation has been most extensively explored in studies of the oral cavity, because it is colonized by hundreds of species of bacteria, making it a likely location for interspecies signaling. Two oral bacteria, Streptococcus gordonii, a commensal species, and Porphyromonas gingivalis, an oral pathogen associated with the progression of periodontitis, are known to form mixed-species biofilms. While $\operatorname{lu} x S$ is dispensable for monospecies biofilm formation in $P$. gingivalis and $S$. gordonii, its expression is required in one of the two species for the mixed biofilm to form (44).

\section{Integration of quorum-sensing information}

The discovery of an autoinducer, AI-2, that is produced and detected by a large number of diverse bacteria implies that bacteria have a means to assess the cell density of other species in a consortium. Many of the bacteria that produce AI-2 also produce and respond to an AHL or an AIP autoinducer, indicating that they also have a mechanism to count their own numbers. While an understanding of how bacteria perceive, integrate, process, and respond appropriately to mixtures of signals is in its infancy, some preliminary studies to identify the molecular underpinnings governing detection and response to combinations of signals have been performed in the model bacterium $V$. harveyi. In $V$. harveyi there are four distinguishable autoinducer states: no autoinducer, AI-1 only, AI-2 only, and AI-1 plus AI-2. By measurement of targetgene expression in response to combinations of these stimuli, it was shown that $V$. harveyi preserves the information from the two binary signals ( \pm AI- 1 and \pm AI-2) and integrates the information into a single channel ( \pm phospho-LuxO) to produce four discrete outputs that correspond to each possible input condition (45). While it is clear from this study that the $V$. harveyi circuit can distinguish between the four input conditions, it was also shown that AI-1 and AI-2 act synergistically (45). This latter result suggests that the $V$. harveyi circuit could function primarily as a coincidence detector that specifically discriminates between the presence of both autoinducers and all other conditions. Studies of the response dynamics of the $V$. harveyi system to various combinations of the two autoinducers should reveal the mechanism that $V$. harveyi uses to accurately distinguish between mixtures containing different concentrations of AI-1 and AI-2. Presumably this will also be the case in other bacteria that respond to multiple autoinducers.

\section{Interference with quorum sensing}

Initial analyses of quorum-sensing systems indicated that they work in a straightforward manner, with bacteria making and responding to a specific signal or combination of signals. However, current research suggests that both prokaryotes and eukaryotes possess interference strategies that they use in natural settings to disrupt bacterial cell-cell communication. In niches where bacteria are competing with other bacterial species for limited nutrients and energy resources, the ability to inactivate or remove an autoinducer or to make an autoinducer antagonist could give one bacterial species an advantage over another that relies on quorum sensing for occupation of a particular niche. Similarly, during infections by pathogenic bacteria that depend on quorum sensing for virulence, the ability of the host to interfere with the signaling process could be the key to staving off infection.

For every class of quorum-sensing signal thus far identified, a mechanism has been discovered that inhibits, destroys, or removes it. For example, Delisea pulchra produces AHL-mediated quorum-sensing inhibitors that promote the degradation of the LuxR class of proteins. A more detailed discussion of this mechanism is provided in this Perspective series by Hentzer and Givskov (46). Enzymes that specifically degrade AHLs have been discovered and shown to quench quorum sensing-controlled behaviors. Two such types of enzymes are now known. One that is produced by Bacillus species is an AHL-lactonase, termed AiiA, which hydrolyzes one of the bonds in the conserved lactone ring present in every tested AHL $(47,48)$. Ring opening inactivates the AHL signals. Introduction of aiiA into several model plants or Caenorhabditis elegans attenuates the virulence of Erwinia carotovora and $P$. aeruginosa. A second enzyme, AiiD, produced by Ralstonia species, is a lactone acylase that cleaves the intact 
lactone ring from the acyl side chain. Again, this action inactivates the signal. When aii D is expressed in $P$. aeruginosa, quorum sensing-regulated functions including swarming motility and virulence factor expression are severely impaired (49). The bacterium Variovorax paradoxus possesses an AHL-degrading activity that appears to be identical in mechanism to that of AiiD (50). V. paradoxus uses AHLs, presumably following separation of the ring from the fatty acid moiety, as a sole source of carbon and nitrogen.

As mentioned above, inhibition of AIP-mediated signaling in Gram-positive bacteria has been well studied in S. aureus. S. aureus strains are categorized into four specificity groups based on the sequence of the thiolactone-containing AIP produced (Figure $2 b)(10,51)$. Each AIP stimulates its own groups' quorum-sensing signal-dependent virulence cascade, while crossinhibiting that of all other groups. Inhibition stems from competitive blocking of the sensor kinase AIPbinding domain by the non-cognate AIP. A group II AIP derivative consisting of only those amino acids that comprise the thiolactone ring functions as a global inhibitor of all four groups. Synthetic AIP derivatives show promising results as inhibitors of virulence in mouse infection models.

Finally, mechanisms that interfere with the luxS/ AI-2-controlled quorum-sensing systems are being discovered. As mentioned above, some bacteria, including the enterics S. typhimurium and E. coli, are able to import extracellular AI-2 into the cell, thus eliminating the signal from the environment (22). This process requires the $\mathrm{ABC}$ transporter encoded by the lsr operon (33). Processing of internalized AI-2 inside the cell renders it nonfunctional as a signal. This action reduces the extracellular AI- 2 concentrations to levels resembling those in a low-cell-density environment. While it is not certain why these bacteria remove AI-2 from their environment, this could be a mechanism that E. coli and S. typhimurium use to "trick" other species into behaving as if they are in a low-density, monospecies environment.

With the discovery and characterization of AI- 2 there is now hope that agonists and antagonists of this universal signal can be identified or synthesized. While inhibitors of AHL and AIP autoinducers may successfully interfere with specific bacterial quorum-sensing systems, an antimicrobial that inhibits LuxS-directed synthesis of DPD or an antagonist of AI-2 itself could well function as a broad-spectrum antibiotic that disrupts quorum-sensing systems in a wide variety of Gram-negative and Gram-positive pathogens. Such therapies may make it possible to control important quorum sensing-regulated functions such as bacterial pathogenesis, horizontal transfer of drug-resistance markers, and biofilm formation. Manipulation of bacterial behaviors using anti-quorum sensing strategies, rather than using antibiotics to kill bacteria, could extend the lifetime of drugs by slowing the emergence of drug-resistant variants.

\section{Conclusions}

The discovery of species-specific as well as universal intercellular signaling molecules reveals that bacteria interact with one another using surprisingly sophisticated mechanisms of communication. In nature, bacteria are rarely found in isolation, and evolution appears to have provided a mechanism that allows them to detect when they are in heterogeneous communities, to assess the proportions of self and other in mixed-species environments, and to respond to this information by appropriately modulating gene expression. Genetic and biochemical studies of the molecular circuitry used for detection of and response to autoinducers, combined with structural identification of the signaling molecules themselves, have enabled us to begin to comprehend how bacteria decipher complex chemical codes. Quorum sensing plays a major role in gene regulation in many environments, and, importantly, convincing data suggest that many pathogens rely on these communication systems to promote infection. Identification of natural strategies or development of synthetic strategies that interfere with intraand interspecies cell-cell communication could provide new treatments for bacterial diseases.

1. Miller, M.B., and Bassler, B.L. 2001. Quorum sensing in bacteria. Annu. Rev. Microbiol. 55:165-199.

2. Engebrecht, J., Nealson, K., and Silverman, M. 1983. Bacterial bioluminescence: isolation and genetic analysis of functions from Vibrio fischeri. Cell. 32:773-781.

3. Kaplan, H.B., and Greenberg, E.P. 1985. Diffusion of autoinducer is involved in regulation of the Vibrio fischeri luminescence system. J. Bacteriol. 163:1210-1214.

4. Fuqua, W.C., Winans, S.C., and Greenberg, E.P. 1994. Quorum sensing in bacteria: the LuxR-LuxI family of cell density-responsive transcriptional regulators. J. Bacteriol. 176:269-275.

5. Lazazzera, B.A., and Grossman, A.D. 1998. The ins and outs of peptide signaling. Trends Microbiol. 6:288-294.

6. Hakenbeck, R., and Stock, J.B. 1996. Analysis of two-component signal transduction systems involved in transcriptional regulation. Methods Enzymol. 273:281-300.

7. Parkinson, J. 1995. Genetic approaches to signaling pathways and proteins. In Two-component signal transduction. J. Hoch and T. Silhavy, editors. ASM Press. Washington, DC, USA. 9-24.

8. Balaban, N., and Novick, R.P. 1995. Autocrine regulation of toxin synthesis by Staphylococcus aureus. Proc. Natl. Acad. Sci. U. S. A. 92:1619-1623.

9. Ji, G., Beavis, R.C., and Novick, R.P. 1995. Cell density control of staphylococcal virulence mediated by an octapeptide pheromone. Proc. Natl. Acad. Sci. U. S. A. 92:12055-12059.

10. Mayville, P., et al. 1999. Structure-activity analysis of synthetic autoinducing thiolactone peptides from Staphylococcus aureus responsible for virulence. Proc. Natl. Acad. Sci. U. S. A. 96:1218-1223.

11. Novick, R.P., et al. 1993. Synthesis of staphylococcal virulence factors is controlled by a regulatory RNA molecule. EMBO J. 12:3967-3975.

12. Rather, P.N., Parojcic, M.M., and Paradise, M.R. 1997. An extracellular factor regulating expression of the chromosomal aminoglycoside $2^{\prime}-\mathrm{N}$ acetyltransferase of Providencia stuartii. Antimicrob. Agents Chemother. 41:1749-1754.

13. Rather, P.N., Ding, X., Baca-DeLancey, R.R., and Siddiqui, S. 1999. Providencia stuartii genes activated by cell-to-cell signaling and identification of a gene required for production or activity of an extracellular factor. J. Bacteriol. 181:7185-7191.

14. Gallio, M., Sturgill, G., Rather, P., and Kylsten, P. 2002. A conserved mechanism for extracellular signaling in eukaryotes and prokaryotes. Proc. Natl. Acad. Sci. U. S. A. 99:12208-12213.

15. Urban, S., Lee, J.R., and Freeman, M. 2001. Drosophila rhomboid-1 defines a family of putative intramembrane serine proteases. Cell. 107:173-182.

16. Bassler, B.L., Wright, M., and Silverman, M.R. 1994. Sequence and function of LuxO, a negative regulator of luminescence in Vibrio harveyi. Mol. Microbiol. 12:403-412.

17. Bassler, B.L., Wright, M., and Silverman, M.R. 1994. Multiple signalling 
systems controlling expression of luminescence in Vibrio harveyi: sequence and function of genes encoding a second sensory pathway. Mol. Microbiol. 13:273-286.

18. Lilley, B.N., and Bassler, B.L. 2000. Regulation of quorum sensing in Vibrio harveyi by LuxO and sigma-54. Mol. Microbiol. 36:940-954.

19. Bassler, B.L., Wright, M., Showalter, R.E., and Silverman, M.R. 1993. Intercellular signalling in Vibrio harveyi: sequence and function of genes regulating expression of luminescence. Mol. Microbiol. 9:773-786.

20. Schauder, S., Shokat, K., Surette, M.G., and Bassler, B.L. 2001. The LuxS family of bacterial autoinducers: biosynthesis of a novel quorum-sensing signal molecule. Mol. Microbiol. 41:463-476.

21. Chen, X., et al. 2002. Structural identification of a bacterial quorumsensing signal containing boron. Nature. 415:545-549.

22. Surette, M.G., Miller, M.B., and Bassler, B.L. 1999. Quorum sensing in Escherichia coli, Salmonella typhimurium, and Vibrio harveyi: a new family of genes responsible for autoinducer production. Proc. Natl. Acad. Sci. U. S. A. 96:1639-1644

23. Schauder, S., and Bassler, B.L. 2001. The languages of bacteria. Genes Dev. 15:1468-1480

24. Freeman, J.A., and Bassler, B.L. 1999. Sequence and function of LuxU: a two-component phosphorelay protein that regulates quorum sensing in Vibrio harveyi. Mol. Microbiol. 31:665-677.

25. Freeman, J.A., Lilley, B.N., and Bassler, B.L. 2000. A genetic analysis of the functions of LuxN: a two-component hybrid sensor kinase that regulates quorum sensing in Vibrio harveyi. Mol. Microbiol. 35:139-149.

26. Martin, M., Showalter, R., and Silverman, M. 1989. Identification of a locus controlling expression of luminescence genes in Vibrio harveyi. J. Bacteriol. 171:2406-2414.

27. Showalter, R.E., Martin, M.O., and Silverman, M.R. 1990. Cloning and nucleotide sequence of luxR, a regulatory gene controlling bioluminescence in Vibrio harveyi. J. Bacteriol. 172:2946-2954.

28. Swartzman, E., Silverman, M., and Meighen, E.A. 1992. The luxR gene product of Vibrio harveyi is a transcriptional activator of the lux promoter. J. Bacteriol. 174:7490-7493.

29. Bassler, B.L., Greenberg, E.P., and Stevens, A.M. 1997. Cross-species induction of luminescence in the quorum-sensing bacterium Vibrio harveyi. J. Bacteriol. 179:4043-4045.

30. Xavier, K.B., and Bassler, B.L. 2003. LuxS quorum sensing: more than just a numbers game. Curr. Opin. Microbiol. 6:191-197.

31. Sperandio, V., Mellies, J.L., Nguyen, W., Shin, S., and Kaper, J.B. 1999. Quorum sensing controls expression of the type III secretion gene transcription and protein secretion in enterohemorrhagic and enteropathogenic Escherichia coli. Proc. Natl. Acad. Sci. U. S. A. 96:15196-15201.

32. DeLisa, M.P., Wu, C.F., Wang, L., Valdes, J.J., and Bentley, W.E. 2001. DNA microarray-based identification of genes controlled by autoinducer 2 stimulated quorum sensing in Escherichia coli.J. Bacteriol. 183:5239-5247.

33. Taga, M.E., Semmelhack, J.L., and Bassler, B.L. 2001. The LuxS-dependent autoinducer AI- 2 controls the expression of an $\mathrm{ABC}$ transporter that functions in AI-2 uptake in Salmonella typhimurium. Mol. Microbiol. 42:777-793

34. Taga, M.E., and Bassler, B.L. 2003. Lsr-mediated transport and processing of AI-2 in Salmonella typhimurium. Mol. Microbiol. In press.

35. Miller, M.B., Skorupski, K., Lenz, D., Taylor, R.K., and Bassler, B.L. 2002. Parallel quorum sensing systems converge to regulate virulence in Vibrio cholerae. Cell. 110:303-314.

36. Hammer, B.H., and Bassler, B.L. 2003. Quorum sensing controls biofilm formation in Vibrio cholerae. Mol. Microbiol. 50:101-114.

37. Zhu, J., et al. 2002. Quorum-sensing regulators control virulence gene expression in Vibrio cholerae. Proc. Natl. Acad. Sci. U. S. A. 99:3129-3134.

38. Finkelstein, R.A., Boesman-Finkelstein, M., Chang, Y., and Hase, C.C. 1992. Vibrio cholerae hemagglutinin/protease, colonial variation, virulence, and detachment. Infect. Immun. 60:472-478.

39. Lyon, W.R., Madden, J.C., Levin, J.C., Stein, J.L., and Caparon, M.G. 2001 Mutation of luxS affects growth and virulence factor expression in Streptococcus pyogenes. Mol. Microbiol. 42:145-157.

40. Ohtani, K., Hayashi, H., and Shimizu, T. 2002. The luxS gene is involved in cell-cell signalling for toxin production in Clostridium perfringens. Mol. Microbiol. 44:171-179.
41. Costerton, J.W., et al. 1994. Biofilms, the customized microniche. J. Bacteriol. 176:2137-2142.

42. Singh, P.K., et al. 2000. Quorum-sensing signals indicate that cystic fibrosis lungs are infected with bacterial biofilms. Nature. 407:762-764.

43. Davies, D.G., et al. 1998. The involvement of cell-to-cell signals in the development of a bacterial biofilm. Science. 280:295-298.

44. McNab, R., et al. 2003. LuxS-based signaling in Streptococcus gordonii: autoinducer 2 controls carbohydrate metabolism and biofilm formation with Porphyromonas gingivalis. J. Bacteriol. 185:274-284.

45. Mok, K.C., Wingreen, N.S., and Bassler, B.L. 2003. Vibrio harveyi quorum sensing: a coincidence detector for two autoinducers controls gene expression. EMBOJ. 22:870-881.

46. Hentzer, M., and Givskov, M. 2003. Pharmacological inhibition of quorum sensing for the treatment of chronic bacterial infections. J. Clin. Invest. 112:1300-1307. doi:10.1172/JCI200320074.

47. Dong, Y.H., Xu, J.L., Li, X.Z., and Zhang, L.H. 2000. AiiA, an enzyme that inactivates the acylhomoserine lactone quorum-sensing signal and attenuates the virulence of Erwinia carotovora. Proc. Natl. Acad. Sci. U. S. A. 97:3526-3531.

48. Dong, Y.H., et al. 2001. Quenching quorum-sensing-dependent bacterial infection by an $\mathrm{N}$-acyl homoserine lactonase. Nature. 411:813-817.

49. Lin, Y.H., et al. 2003. Acyl-homoserine lactone acylase from Ralstonia strain XJ12B represents a novel and potent class of quorum-quenching enzymes. Mol. Microbiol. 47:849-860.

50. Leadbetter, J.R., and Greenberg, E.P. 2000. Metabolism of acyl-homoserine lactone quorum-sensing signals by Variovorax paradoxus. J. Bacteriol. 182:6921-6926

51. Ji, G., Beavis, R., and Novick, R.P. 1997. Bacterial interference caused by autoinducing peptide variants. Science. 276:2027-2030.

52. Fong, K.P., Gao, L., and Demuth, D.R. 2003. luxS and $\operatorname{arcB}$ control aerobic growth of Actinobacillus actinomycetemcomitans under iron limitation. Infect. Immun. 71:298-308.

53. Fong, K.P., Chung, W.O., Lamont, R.J., and Demuth, D.R. 2001. Intraand interspecies regulation of gene expression by Actinobacillus actinomycetemcomitans LuxS. Infect. Immun. 69:7625-7634.

54. Stevenson, B., and Babb, K. 2002. LuxS-mediated quorum sensing in Borrelia burgdorferi, the lyme disease spirochete. Infect. Immun. 70:4099-4105.

55. Elvers, K.T., and Park, S.F. 2002. Quorum sensing in Campylobacter jejuni: detection of a luxS encoded signalling molecule. Microbiology. 148: $1475-1481$

56. Sperandio, V., Torres, A.G., Giron, J.A., and Kaper, J.B. 2001. Quorum sensing is a global regulatory mechanism in enterohemorrhagic Escherichia coli O157:H7. J. Bacteriol. 183:5187-5197.

57. Winzer, K., et al. 2002. Role of Neisseria meningitidis luxS in cell-to-cell signaling and bacteremic infection. Infect. Immun. 70:2245-2248.

58. Derzelle, S., Duchaud, E., Kunst, F., Danchin, A., and Bertin, P. 2002. Identification, characterization, and regulation of a cluster of genes involved in carbapenem biosynthesis in Photorhabdus luminescens. Appl. Environ. Microbiol. 68:3780-3789.

59. Burgess, N.A., et al. 2002. LuxS-dependent quorum sensing in Porphyromonas gingivalis modulates protease and haemagglutinin activities but is not essential for virulence. Microbiology. 148:763-772.

60. Chung, W.O., et al. 2001. Signaling system in Porphyromonas gingivalis based on a LuxS protein. J. Bacteriol. 183:3903-3909.

61. Prouty, A.M., Schwesinger, W.H., and Gunn, J.S. 2002. Biofilm formation and interaction with the surfaces of gallstones by Salmonella spp. Infect. Immun. 70:2640-2649.

62. Day, W.A., Jr., and Maurelli, A.T. 2001. Shigella flexneri LuxS quorum-sensing system modulates virB expression but is not essential for virulence. Infect. Immun. 69:15-23.

63. Merritt, J., Qi, F., Goodman, S.D., Anderson, M.H., and Shi, W. 2003. Mutation of luxS affects biofilm formation in Streptococcus mutans. Infect. Immun. 71:1972-1979.

64. Stroeher, U.H., Paton, A.W., Ogunniyi, A.D., and Paton, J.C. 2003. Mutation of luxS of Streptococcus pneumoniae affects virulence in a mouse model. Infect. Immun. 71:3206-3212.

65. Kim, S.Y., et al. 2003. Regulation of Vibrio vulnificus virulence by the LuxS quorum-sensing system. Mol. Microbiol. 48:1647-1664. 